Journal of Applied Veterinary Sciences, 6 (3): 76 -85 ( July, 2021).

ISSN: Online: 2090-3308, Print: 1687-4072

Journal homepage : https://javs.journals.ekb.eg

\title{
The Effect of Feeding Frequency and Amount on Performance, Behavior and Physiological Responses of Broilers
}

\author{
Enas K. Aziz ${ }^{1 *}$ and Shimaa R. Masoud ${ }^{2}$
}

${ }^{1}$ Department of Husbandry and Animal Wealth Development, Animal Behavior and management, Faculty of Veterinary Medicine, University of Sadat City, Egypt.

${ }^{2}$ Department of Physiology, Faculty of Veterinary Medicine, University of Sadat City, Egypt.

*Corresponding Author, Enas K. Aziz; E-mail: enas.aziz@vet.usc.edu.eg

\section{ABSTRACT}

The aim of this study was to conclude the effect of feed frequencies and amount on growth performance, carcass traits, physiological responses and behaviors of broilers chicks. One hundred Ross broiler chicks at 7 days old were randomly assigned into five regimens (20 chicks per each program with two replicate). Experimental design were as follows; T1: feeding once a day (100\% diet at 6:30), T2: two equal feedings per day (50\% of total diet at 6:30 and 18:30), T3: two feedings per day $(75 \%$ at 6:30 and $25 \%$ at 18:30), T4: three equal feedings per day $(33.33 \%$ at $6: 30$, $12: 30$ and 18:30) and T5: three feedings per day as $50 \%$ of diet at $6: 30,25 \%$ at $12: 30$ and $25 \%$ at 18:30. Chicks were fed with commercial broiler diet for five weeks. The obtained results indicated that offering two equal feeding times per day was significant $(\mathrm{p}<0.05)$ improved overall body weight, body weight gain and average daily gain of broiler chicks. Chicks fed single, twice per day and three times of $4^{\text {th }}$ regimen showed higher feed intake than chicks of $3^{\text {rd }}$ and $5^{\text {th }}$ regimens. Feed frequencies had significant $(\mathrm{P}<0.05)$ effect on percent of carcass, spleen and intestine percentage. High proportion of chicks of $1^{\text {st }}, 2^{\text {nd }}, 3^{\text {rd }}$, and $4^{\text {th }}$ feeding regimens showed significant more feeding and preening behaviors than chicks of $5^{\text {th }}$ regimen. Chicks of $5^{\text {th }}$ regimen exhibited significantly higher foraging behavior than those of $3^{\text {rd }}$ and $4^{\text {th }}$ regimens. Moreover, feeding frequency with different amount of feed decreased the duration of tonic immobility test means less stressful and high chick welfare. T2 showed significant increasing on heterophil, total protein, globulin, serum glucose and growth hormone levels in comparison to the other groups, as well as a lower albumin/globulin ratio and albumin. In conclusion, two equal feeding per day (T2) might be a desirable feeding approach that might produce good healthy birds with maximum growth performance and improved welfare.
\end{abstract}

Keywords: Behaviour, Broilers, Feeding frequency, Growth performance, Physiology.

\section{INTRODUCTION}

The productivity of broiler industry can be successfully realized with improved growth performance, feed efficiency and health of birds (Baracho et al., 2006). Feed management considered the most expenditure in broiler management account (Neves et al., 2014). Consequently, efficiency in feeding is one of the fundamental factors for successful poultry production.
Original Article:

DOI:https://dx.doi.org/10.21608/javs.2 $\underline{021.80537 .1086}$

Received :11 June, 2021.

Accepted :14 July, 2021.

Published in July, 2021.

This is an open access article under the term of the Creative Commons Attribution 4.0 (CC-BY) International License . To view a copy of this license, visit:

http://creativecommons.org/licenses/by/4.0/

J. Appl. Vet. Sci., 6(3): $76-85$.

Domestic birds consume their food regularly throughout the day and do not eat separate meals (Peter and Gernat, 2006). In poultry production, ad libitum feeding is widely used for maximizing bird growth. Additionally, allowing birds an indefinite supply of feed may effect bird consumption that exceeds its requirements for maintenance and production as well as the excessive deposition of body fat at market age that reduces meat quality (Junqueira et al., 2003; Butzen et al., 2013) and increases the 


\section{Enas K. Aziz and Shimaa R. Masoud}

incidence of sudden death syndrome, ascites, and skeletal problems (Khurshid et al., 2019).

When birds forced on specific meal times or regimes an adaptation is observed as birds consume an amount of feed in a shorter time. Thus, the desirable feeding approach would be to produce chickens with maximum lean body mass, minimum feed intake, and extreme final body weight (Peter and Gernat, 2006). One management strategy to reduce fat deposition and prevent metabolic troubles is feed restriction manner (Mohammadalipour et al., 2017).

Feeding frequency is one of the feed restriction strategy. In broiler chickens, restricted feeding frequency has been used to decrease feed consumption and increase feed efficiency (Farghly and Makled, 2015). Intermittent feeding regimes ( $4 \mathrm{~h}$ of feeding and $4 \mathrm{~h}$ of non-feeding) had higher average daily gain and lower feed conversion ratio values than restricted feeding regimens and did not produce any negative effects on performance or physiological status of birds (Farghly et al., 2019). Liu et al., (2020) concluded that both ad libitum feeding and fixed feeding frequency for 3 to 5 times daily were suitable for geese from 28 to 70 day of age to achieve optimum production. In sucking piglets, 6 times daily feedings led to a higher average daily gain and lower feed conversion ratio than those in pigs fed 12 times daily (Liu et al., 2019).

Without a thorough blood examination, it is frequently difficult to determine an animal's present health status (Amakir $\boldsymbol{e t}$ al., 2009). The existence of many metabolites and other constituents in the body can be clinically investigated using blood pictures, and blood plays an important part in the animal's physiological, nutritional, and pathological status (Aderemi, 2004). In the poultry industry, blood parameters have become increasingly popular as a tool for detecting physiological changes and aiding illness detection. Hematological markers are important indicators of a bird's physiological status (Saied $\boldsymbol{e t}$ al., 2011).

The evaluation of normal hematological indices of broiler chickens is crucial for detecting various clinical and/or metabolic disorders. This can be used to assess the health of a single bird or a flock of birds (Olukomaiya et al., 2014). The level of a specific blood ingredient can be influenced by many factors such as feeding, environment, rearing technique, and physiological state (Meluzzi et al., 1992). Increasing animal wellbeing can occasionally result in the systemic immune system being suppressed, resulting in welfare problems triggered by aberrant behaviors (De Jong et al., 2003). Feed limitation improved the immunological response of broilers, which was harmed by heat stress exposure
(Khajavi et al., 2003). Thus, the aim of this study was to investigate the effect of feeding amount and frequency on growth performance, carcass traits, behavior and physiological responses of broilers from 7 days till marketing age.

\section{MATERIAL AND METHODS}

All birds handling procedures as well as samples collection and disposal were according to the regulation of institutional Animal Care and Use Committee (IACUC) Faculty of Veterinary Medicine, University of Sadat City, Cairo, Egypt during the period from September 2020 to November 2020.

Five feeding regimens were applied for 100 Ross broiler chicks at 7 day old (20 chicks per each regimen in 2 replicate) as it is shown in Table (1). These programs last for 5 weeks until chicks reach to 40 days old. Feed was provided ad libitum for the first 7 days of chick life. At 7 days the chicks were divided into five treatments and were housed in separated pens (10 chicks/ pen) constructed on the concrete floor with wire partitioning (floor system) as described by (Abdelbasit et al., 2016). The house was equipped with feeders and drinkers and wood shaving was used as the litter material under continuous lighting system. Chicks were fed on commercial broiler starter $(23 \%$ crude protein) for first 2 weeks, grower $(21.5 \%$ crude protein) form 2 till 4 weeks and finisher (19\% crude protein) from 4 weeks until slaughter. Daily feed allowance was adjusted according to the recommendation made by an Aviagen brand 2019. Water was provided ad libitum. All birds were vaccinated against Newcastle disease at 8 and 18 day of chick age in drinking water. All general management practices were followed until the birds reach to 40 days.

Table 1: Experimental design

\begin{tabular}{|c|c|c|c|c|c|c|}
\hline \multirow{3}{*}{$\begin{array}{l}\text { Feeding } \\
\text { regimens }\end{array}$} & \multicolumn{6}{|c|}{ Feeding amount and time } \\
\hline & \multicolumn{2}{|c|}{ First meal } & \multicolumn{2}{|c|}{ Second meal } & \multicolumn{2}{|c|}{ Third meal } \\
\hline & $\begin{array}{l}\vec{D} \\
0 \\
\stackrel{0}{E} \\
\text { dq }\end{array}$ & $\Xi$ & 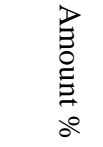 & $\bar{\Xi}$ & 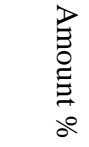 & $\overline{\mathrm{E}}$ \\
\hline T1 & 100 & $6: 30$ & - & - & - & - \\
\hline $\mathrm{T} 2$ & 50 & $6: 30$ & 50 & $18: 30$ & - & - \\
\hline T3 & 75 & $6: 30$ & 25 & $18: 30$ & - & - \\
\hline $\mathrm{T} 4$ & 33.33 & $6: 30$ & 33.33 & $12: 30$ & 33.33 & $18: 30$ \\
\hline T5 & 50 & $6: 30$ & 25 & $12: 30$ & 25 & $18: 30$ \\
\hline
\end{tabular}

Data Collection:

Growth Performance:

Body weight of chicks was measured at 7, 21, 30 and 40 days old. Moreover, weight gain was calculated as difference between final body weight and initial body weight. Additionally average daily gain 
and feed conversion ratio (FCR) (feed intake (g)/weight gain (g)) were calculated. Daily feed intake was calculated daily by measuring given feed and remained feed per treatment.

\section{Behavioral observation:}

Scanning technique of observations was performed according to Fraser and Broom, (1990) for three consecutive weeks (3th, $4^{\text {th }}$, and $5^{\text {th }}$ week). All birds of each treatment were observed 2 days/week for 10 minutes/time in two observational periods; in the morning (8.00-8:50) and at afternoon (17:00- 17:50). The percentage of chicks performed feeding, drinking, foraging, lying, standing, walking, preening, wing flapping and stretching behaviors was recorded during all scan samples in each treatment.

\section{Tonic immobility:}

The effect of the feeding frequency and amount on the fear response of the chicks was evaluated with the tonic immobility test (TI). A total of 5 chicks per treatment were used for this test at 14, 28 and 40 days of age. The test was performed between 15:00 and 17:00 $\mathrm{h}$ in a separate room away from chick's pen. Tonic immobility was induced by placing the bird on its back on a U-shaped wooden cradle padded with a layer of cloth according to Tahamtani and Riber, (2020) for maximum $5 \mathrm{~min}$ test period, by stop watch, and recorded the total duration of TI, i.e. until the bird righted itself.

\section{Carcass traits:}

Five birds were randomly selected from each treatment at $40^{\text {th }}$ days of age and they were slaughtered. The weights of carcass and internal organs (liver, heart, gizzard, spleen, bursa and intestine) were taken and expressed as percentage of the live weight at slaughter.

\section{Blood samples and Biochemical Assays:}

Four blood samples were obtained from each chick's wing vein at 40 days old and divided into two parts one in a vial containing EDTA for hematological assay and another part for serum separation. In the laboratory, blood samples were subjected to hematological examination of hemoglobin concentration $(\mathrm{Hb})$, Red Blood Cell (RBC), White Blood Cell (WBC), Packed Cell Volume (PCV), mean corpuscular volume (MCV), mean corpuscular hemoglobin $(\mathrm{MCH})$, mean corpuscular hemoglobin concentration (MCHC) and White Blood Cell differential counts according to conventional techniques according to Schalm, (1986). In addition, total protein, albumin, and globulin were measured in serum, as recommended by electrophoresis. Glucose assay kit (Diamond diagnostic kits) was used to detect the levels of glucose in the serum. Measurement of chicken growth hormone (CGH) in serum samples using a Recombinant Chicken GH (BioVision Rsearch Products).

\section{Statistical analysis:}

Statistical analysis was carried out using analysis of variance; one-way ANOVA test followed by Duncan multiple comparison tests. All data were statistically analyzed using statistical software program SPSS (Statistical package for Social Sciences) Version 22. The results were expressed as mean $\pm \mathrm{SE}$ and considered as significant when $P$ - values less than 0.05 $(*)$ and $0.01(* *)$.

\section{Growth performance:}

Data presented in Table (2) revealed that feeding frequency with different amount had a significant effect on growth performance of broiler. Generally, offering two equal feeding per day had a significant $(p<0.05)$ effect on overall body weight, body weight gain and average daily gain of broiler chicks. Body weight at 21 and 30 days of chicks age were significantly $(p<0.01)$ improved under two equal feeding of $2^{\text {nd }}$ program (T2) and three feeding of $5^{\text {th }}$ program (T5) compared to $3^{\text {rd }}$ feeding programs (T3) only. However, offering feed for broiler chicks once and twice per day with equal amount had a significant $(p<0.05)$ effect on body weight of chicks at 40 days old. The FCR of chicks is not significantly affected by different feeding programs.

Data in Table (3) showed that, there is a significant difference in feed intake among all groups. Overall feed intake of chicks of T1, T2 and T4 was significantly higher than chicks of T3. At $1^{\text {st }}$ and $2^{\text {nd }}$ week, chicks of $2^{\text {nd }}$ program (T2) showed a significant increase feed intake than chicks of $5^{\text {th }}$ program (T5). However chicks fed single, twice per day and three times of $4^{\text {th }}$ program showed higher feed intake at $3^{\text {rd }}$ and $4^{\text {th }}$ weeks than chicks of $3^{\text {rd }}$ and $5^{\text {th }}$ program.

\section{Behavioral observation and tonic immobility test (TI):}

The effects of different feeding programs on behaviors of broiler were presented in Table (4). High proportion of chicks of $1^{\text {st }}, 2^{\text {nd }}, 3^{\text {rd }}$, and $4^{\text {th }}$ feeding regimens were showed significant more feeding behavior than chicks of $5^{\text {th }}$ program. Chicks of $5^{\text {th }}$ program were exhibited significantly higher foraging behavior than those of $3^{\text {rd }}$ and $4^{\text {th }}$ program. Chicks of $1^{\text {st }}, 2^{\text {nd }}$, and $4^{\text {th }}$ feeding regimens were significantly showed higher preening behavior than those of $5^{\text {th }}$ program. Conversely, other behavioral patterns of chicks including drinking, resting, walking, standing, wing flapping and stretching was not significantly differed under different feeding programs. Results summarized in Table (5) concluded that feeding frequency with different amount of feed had a significant $(P<0.05)$ effect on the duration of tonic immobility test (TI). The chicks of $1^{\text {st }}$ regimen that fed one time per day were showed significantly longer duration in TI than other regimens that indicated increasing the fear levels. 


\section{Enas K. Aziz and Shimaa R. Masoud \\ RESULTS}

Table 2: Effect of feeding frequency and amount on body weight (g), weight gain (g) ,average daily gain $(\mathrm{g})$ and feed conversion ratio (FCR) of broiler (Means \pm SE)

\begin{tabular}{|c|c|c|c|c|c|c|}
\hline \multirow{2}{*}{ Items } & \multicolumn{5}{|c|}{ Feeding regimens } & \multirow{2}{*}{$\begin{array}{c}P \\
\text { value }\end{array}$} \\
\hline & $\mathrm{T} 1$ & $\mathrm{~T} 2$ & T3 & $\mathrm{T} 4$ & $\mathrm{T5}$ & \\
\hline \multicolumn{7}{|l|}{ Body weight $(\mathrm{g})$} \\
\hline at 7 days & $\begin{array}{c}146.81 \pm \\
3.29\end{array}$ & $\begin{array}{c}146.99 \pm \\
3.00\end{array}$ & $\begin{array}{c}145.32 \pm \\
2.31\end{array}$ & $\begin{array}{c}149.73 \pm \\
3.32\end{array}$ & $\begin{array}{c}147.62 \pm \\
2.53\end{array}$ & NS \\
\hline at 21 days & $\begin{array}{c}855.48 \pm \\
8.70^{\mathrm{ab}}\end{array}$ & $\begin{array}{c}894.81 \pm \\
5.50^{\mathrm{a}}\end{array}$ & $\begin{array}{c}825.50 \pm \\
1.31^{\mathrm{b}}\end{array}$ & $\begin{array}{c}854.78 \pm \\
1.88^{\mathrm{ab}}\end{array}$ & $\begin{array}{c}889.79 \pm \\
7.33^{\mathrm{a}}\end{array}$ & $* *$ \\
\hline at 30 days & $\begin{array}{c}1728.57 \pm \\
6.75^{\mathrm{ab}}\end{array}$ & $\begin{array}{c}1769.23 \pm \\
3.96^{\mathrm{a}}\end{array}$ & $\begin{array}{c}1618.75 \pm \\
2.87^{\mathrm{b}}\end{array}$ & $\begin{array}{c}1700.63 \pm \\
3.85^{\mathrm{ab}}\end{array}$ & $\begin{array}{c}1785.00 \pm \\
2.33^{\mathrm{a}}\end{array}$ & $* *$ \\
\hline at 40 days & $\begin{array}{c}2004.16 \pm \\
2.55^{\mathrm{a}}\end{array}$ & $\begin{array}{c}1999.50 \pm \\
3.36^{\mathrm{a}}\end{array}$ & $\begin{array}{c}1880.00 \pm \\
3.50^{\mathrm{b}}\end{array}$ & $\begin{array}{c}1963.00 \pm \\
3.26^{\mathrm{ab}}\end{array}$ & $\begin{array}{c}1986.87 \pm \\
2.01^{\mathrm{ab}}\end{array}$ & * \\
\hline $\begin{array}{l}\text { Overall weight } \\
(\mathrm{g})\end{array}$ & $\begin{array}{c}1143.48 \pm \\
6.75^{\mathrm{ab}}\end{array}$ & $\begin{array}{c}1203.48 \pm \\
6.16^{\mathrm{a}}\end{array}$ & $\begin{array}{c}1079.93 \pm \\
5.19^{\mathrm{b}}\end{array}$ & $\begin{array}{c}1136.70 \pm \\
6.75^{\mathrm{ab}}\end{array}$ & $\begin{array}{c}1040.03 \pm \\
6.05^{\mathrm{b}}\end{array}$ & $*$ \\
\hline $\begin{array}{l}\text { Body weight } \\
\text { gain (g/bird) }\end{array}$ & $\begin{array}{c}1856.05 \pm \\
2.12^{\mathrm{ab}}\end{array}$ & $\begin{array}{c}1921.78 \pm \\
3.87^{\mathrm{a}}\end{array}$ & $\begin{array}{c}1799.71 \pm \\
3.41^{\mathrm{b}}\end{array}$ & $\begin{array}{c}1815.14 \pm \\
2.58^{\mathrm{ab}}\end{array}$ & $\begin{array}{c}1779.92 \pm \\
3.25^{\mathrm{b}}\end{array}$ & $*$ \\
\hline $\begin{array}{l}\text { Average daily } \\
\text { gain }(\mathrm{g})\end{array}$ & $\begin{array}{c}44.17 \pm \\
1.26^{\mathrm{b}}\end{array}$ & $\begin{array}{c}47.04 \pm \\
0.71^{\mathrm{a}}\end{array}$ & $\begin{array}{c}44.09 \pm \\
0.67^{\mathrm{b}}\end{array}$ & $\begin{array}{c}44.49 \pm \\
0.67^{\mathrm{b}}\end{array}$ & $\begin{array}{c}44.71 \pm \\
0.38^{\mathrm{b}}\end{array}$ & $*$ \\
\hline FCR & $\begin{array}{c}2.84 \pm \\
0.22\end{array}$ & $\begin{array}{c}2.81 \pm \\
0.22\end{array}$ & $\begin{array}{c}2.56 \pm \\
0.12\end{array}$ & $\begin{array}{c}2.65 \pm \\
0.08\end{array}$ & $\begin{array}{c}2.52 \pm \\
0.09\end{array}$ & NS \\
\hline
\end{tabular}

$\mathrm{a}, \mathrm{b},=$ Mean values within rows with different superscripts letters are significantly different $*(p<0.05)$ or $* *(p<0.01)$, NS non significant.

Table 3: Effect of feeding frequency and amount on feed intake ( $\mathrm{g} / \mathrm{bird})$ of broiler (Means $\pm \mathrm{SE}$ ).

\begin{tabular}{|c|c|c|c|c|c|c|}
\hline \multirow{2}{*}{$\begin{array}{l}\text { Feed intake } \\
\text { (g/bird) }\end{array}$} & \multicolumn{5}{|c|}{ Feeding regimens } & \multirow{2}{*}{$\begin{array}{l}P \\
\text { value }\end{array}$} \\
\hline & $\mathrm{T} 1$ & $\mathrm{~T} 2$ & $\mathrm{~T} 3$ & $\mathrm{~T} 4$ & T5 & \\
\hline At $1^{\text {st }}$ week & $\begin{array}{l}46.54 \pm \\
2.25^{\mathrm{ab}}\end{array}$ & $\begin{array}{c}48.09 \pm \\
2.17^{\mathrm{a}}\end{array}$ & $\begin{array}{c}45.80 \pm \\
1.83^{\mathrm{ab}}\end{array}$ & $\begin{array}{c}44.12 \pm \\
1.96^{\mathrm{ab}}\end{array}$ & $\begin{array}{c}40.71 \pm \\
1.12^{\mathrm{b}}\end{array}$ & $*$ \\
\hline At $2^{\text {nd }}$ week & $\begin{array}{c}80.83 \pm \\
3.64^{\mathrm{ab}}\end{array}$ & $\begin{array}{c}83.51 \pm \\
2.13^{\mathrm{a}}\end{array}$ & $\begin{array}{c}79.86 \pm \\
2.43^{\mathrm{ab}}\end{array}$ & $\begin{array}{c}80.28 \pm \\
2.30^{\mathrm{ab}}\end{array}$ & $\begin{array}{c}72.59 \pm \\
2.61^{\mathrm{b}}\end{array}$ & $*$ \\
\hline At $3^{\text {rd }}$ week & $\begin{array}{c}124.97 \pm \\
2.62^{\mathrm{a}}\end{array}$ & $\begin{array}{c}124.23 \pm \\
2.72^{\mathrm{a}}\end{array}$ & $\begin{array}{c}116.12 \pm \\
2.94^{\mathrm{b}}\end{array}$ & $\begin{array}{c}123.32 \pm \\
2.56^{\mathrm{a}}\end{array}$ & $\begin{array}{c}114.39 \pm \\
2.50^{\mathrm{b}}\end{array}$ & $* *$ \\
\hline At $4^{\text {th }}$ week & $\begin{array}{c}165.35 \pm \\
4.36^{\mathrm{a}}\end{array}$ & $\begin{array}{c}164.66 \pm \\
3.37^{\mathrm{a}}\end{array}$ & $\begin{array}{c}158.36 \pm \\
3.17^{\mathrm{b}}\end{array}$ & $\begin{array}{c}162.61 \pm \\
2.43^{\mathrm{a}}\end{array}$ & $\begin{array}{c}155.19 \pm \\
2.71^{\mathrm{b}}\end{array}$ & $*$ \\
\hline Overall & $\begin{array}{c}105.33 \pm \\
4.32^{\mathrm{a}}\end{array}$ & $\begin{array}{c}104.47 \pm \\
4.41^{\mathrm{a}}\end{array}$ & $\begin{array}{c}97.76 \pm \\
4.44^{\mathrm{b}}\end{array}$ & $\begin{array}{c}105.13 \pm \\
6.39^{\mathrm{a}}\end{array}$ & $\begin{array}{c}100.76 \pm \\
4.29^{\mathrm{ab}}\end{array}$ & $*$ \\
\hline
\end{tabular}

$\mathrm{a}, \overline{\mathrm{b},}=$ Mean values within rows with different superscripts letters are significantly different $*(p<0.05)$ or $* *(p<0.01)$.

Table 4: Effect of feeding frequency and amount on behaviors (\%) of broiler (Means \pm SE).

\begin{tabular}{|c|c|c|c|c|c|c|}
\hline \multirow[t]{2}{*}{ Behaviour } & \multicolumn{5}{|c|}{ Feeding regimens } & \multirow[t]{2}{*}{$P$ value } \\
\hline & $\mathrm{T} 1$ & $\mathrm{~T} 2$ & $\mathrm{~T} 3$ & $\mathrm{~T} 4$ & $\mathrm{~T} 5$ & \\
\hline Feeding & $34.10 \pm 2.39^{\mathrm{a}}$ & $39.47 \pm 1.80^{\mathrm{a}}$ & $36.47 \pm 2.41^{\mathrm{a}}$ & $36.28 \pm 2.99^{\mathrm{a}}$ & $23.75 \pm 3.15^{\mathrm{b}}$ & $* *$ \\
\hline Drinking & $18.65 \pm 1.12$ & $17.34 \pm 1.49$ & $18.31 \pm 1.48$ & $18.24 \pm 1.75$ & $18.21 \pm 2.02$ & NS \\
\hline Foraging & $16.56 \pm 0.95^{\mathrm{ab}}$ & $16.44 \pm 1.03^{\mathrm{ab}}$ & $14.80 \pm 1.40^{\mathrm{b}}$ & $13.34 \pm 1.42^{\mathrm{b}}$ & $18.82 \pm 1.32^{\mathrm{a}}$ & $*$ \\
\hline Lying & $32.46 \pm 1.67$ & $33.16 \pm 1.68$ & $32.56 \pm 2.45$ & $30.31 \pm 2.72$ & $32.98 \pm 2.64$ & NS \\
\hline Walking & $13.73 \pm 1.34$ & $13.98 \pm 0.97$ & $13.93 \pm 0.82$ & $12.64 \pm 1.31$ & $13.70 \pm 1.86$ & NS \\
\hline Standing & $14.39 \pm 1.13$ & $15.85 \pm 2.21$ & $15.18 \pm 1.05$ & $14.33 \pm 1.47$ & $14.16 \pm 1.31$ & NS \\
\hline Wing flapping & $12.47 \pm 0.79$ & $11.81 \pm 0.50$ & $14.74 \pm 1.80$ & $12.90 \pm 1.76$ & $12.76 \pm 1.38$ & NS \\
\hline Stretching & $13.13 \pm .89$ & $13.26 \pm .67$ & $14.12 \pm 1.54$ & $14.70 \pm 2.10$ & $12.81 \pm 1.23$ & NS \\
\hline Preening & $17.78 \pm 1.22^{\mathrm{a}}$ & $17.81 \pm 1.27^{\mathrm{a}}$ & $15.55 \pm 1.20^{\mathrm{ab}}$ & $19.25 \pm 2.15^{\mathrm{a}}$ & $13.54 \pm 1.44^{\mathrm{b}}$ & $*$ \\
\hline
\end{tabular}

$\mathrm{a}, \mathrm{b},=$ Mean values within rows with different superscripts letters are significantly different $*(p<0.05)$ or $* *(p<0.01)$, NS non significant. 


\section{The Effect of Feeding Frequency and Amount on Performance. ......}

Table 5: Effect of feeding frequency and amount on Tonic Immobility (TI) duration (sec) of broiler (Means \pm SE).

\begin{tabular}{ccccccc}
\hline \multirow{2}{*}{$\begin{array}{c}\text { TI duration } \\
\text { (sec) }\end{array}$} & \multicolumn{5}{c}{ Feeding regimens } & \begin{tabular}{l}
$P$ \\
valu \\
\cline { 2 - 7 }
\end{tabular} \\
\cline { 2 - 7 } & $\mathrm{T} 1$ & $\mathrm{~T} 2$ & $\mathrm{~T} 3$ & $\mathrm{~T} 4$ & $\mathrm{~T} 5$ & $*$ \\
\hline At 14 d & $73.80 \pm 2.01^{\mathrm{a}}$ & $59.20 \pm 1.77^{\mathrm{ab}}$ & $46.40 \pm 3.61^{\mathrm{b}}$ & $49.00 \pm 2.44^{\mathrm{b}}$ & $48.00 \pm 4.87^{\mathrm{b}}$ & $*$ \\
At 28 d & $67.60 \pm 8.06^{\mathrm{a}}$ & $48.60 \pm 5.24^{\mathrm{b}}$ & $50.60 \pm 10.03^{\mathrm{b}}$ & $46.20 \pm 10.09^{\mathrm{b}}$ & $46.40 \pm 7.67^{\mathrm{b}}$ & $*$ \\
At 40 d & $58.80 \pm 2.57^{\mathrm{a}}$ & $40.60 \pm 3.48^{\mathrm{b}}$ & $47.20 \pm 4.85^{\mathrm{b}}$ & $46.00 \pm 2.60^{\mathrm{b}}$ & $42.60 \pm 2.67^{\mathrm{b}}$ & $*$ \\
\hline \multicolumn{2}{c}{ a, b, $=$ Mean values within rows with different superscripts letters are significantly different $*(p<0.05)}$.
\end{tabular}

\section{Carcass traits:}

Results summarized in Table (6) denoted that feeding frequency with different affect significantly $(p<0.05)$ on carcass traits. Percentage of carcass weight of chicks fed once and twice per day with equal amount was significantly increased compared with carcass of other treatments. Additionally percentage of spleen weight of chicks of the $2 \mathrm{nd}$, $3 \mathrm{rd}$ and $5^{\text {th }}$ showed significant increase compared to the 4th feeding regimens. However intestinal percentage weight of chicks of the T4 was significantly increased than the T1 and T2. Others carcass traits as percentage weight of liver, heart, gizzard and bursa were not significantly differed under feeding frequency regimens.

Table 6: Effect of feeding frequency and amount on carcass traits (\%) of broiler (Means \pm SE).

\begin{tabular}{|c|c|c|c|c|c|c|}
\hline \multirow{2}{*}{$\begin{array}{l}\text { Carcass } \\
\text { traits }(\%)\end{array}$} & \multicolumn{5}{|c|}{ Feeding regimens } & \multirow{2}{*}{$\begin{array}{l}P \\
\text { value }\end{array}$} \\
\hline & $\mathrm{T} 1$ & $\mathrm{~T} 2$ & $\mathrm{~T} 3$ & $\mathrm{~T} 4$ & T5 & \\
\hline Carcass \% & $76.10 \pm 0.82^{\mathrm{a}}$ & $75.90 \pm 1.25^{\mathrm{a}}$ & $71.83 \pm 0.71^{b}$ & $72.47 \pm 0.82^{b}$ & $71.42 \pm 0.96^{b}$ & $*$ \\
\hline Liver \% & $2.63 \pm 0.09$ & $2.62 \pm 0.07$ & $2.56 \pm 0.14$ & $2.52 \pm 0.15$ & $2.54 \pm 0.09$ & NS \\
\hline Heart \% & $0.55 \pm 0.02$ & $0.57 \pm 0.02$ & $0.56 \pm 0.03$ & $0.57 \pm 0.02$ & $0.59 \pm 0.05$ & NS \\
\hline Gizzard \% & $1.93 \pm 0.08$ & $1.81 \pm 0.06$ & $1.72 \pm 0.12$ & $2.22 \pm 0.27$ & $2.01 \pm 0.12$ & NS \\
\hline Spleen \% & $0.15 \pm 0.01^{\mathrm{ab}}$ & $0.17 \pm 0.01^{\mathrm{a}}$ & $0.17 \pm 0.02^{\mathrm{a}}$ & $0.11 \pm 0.02^{b}$ & $0.17 \pm 0.01^{\mathrm{a}}$ & $*$ \\
\hline Bursa $\%$ & $0.15 \pm 0.02$ & $0.16 \pm 0.01$ & $0.15 \pm 0.02$ & $0.13 \pm 0.28$ & $0.14 \pm 0.01$ & NS \\
\hline Intestine \% & $7.76 \pm 0.17^{\mathrm{b}}$ & $7.89 \pm 0.55^{b}$ & $8.41 \pm 0.57^{\mathrm{ab}}$ & $9.76 \pm 0.46^{\mathrm{a}}$ & $8.85 \pm 0.23^{a b}$ & $*$ \\
\hline
\end{tabular}

\section{Physiological responses:}

Table (7) showed that feeding frequency and amount had a significant $(p<0.01)$ effect on heterophil in the second feeding regimen compared to the other groups. According to the results of this study, there was no significant difference $(p>0.05)$ between the groups on hematological parameters as $\mathrm{Hb}, \mathrm{PCV}, \mathrm{RBCs}, \mathrm{MCH}$, and MCV. However chicks of T4 and T5 showed higher MCHC than chicks of T1. Table (8) showed the effect of different feeding frequencies and amounts on broiler serum biochemical testing. The second regimen's chicks had significantly higher total protein, globulin, and gamma globulin levels than the other programs, as well as a lower $\mathrm{A} / \mathrm{G}$ ratio and albumin. The results listed in Table (9) revealed that offering feed to chicks twice daily with equal amount improved serum glucose and growth hormone than others regimens.

Table 7: Effect of feeding frequency and amount on physiological responses of broiler (Means $\pm \mathrm{SE})$.

\begin{tabular}{|c|c|c|c|c|c|c|}
\hline \multirow[t]{2}{*}{ Items } & \multicolumn{5}{|c|}{ Feeding regimens } & \multirow[t]{2}{*}{$P$ value } \\
\hline & T1 & $\mathrm{T} 2$ & T3 & $\mathrm{T} 4$ & T5 & \\
\hline $\mathrm{WBC} \times 10^{3} / \mu \mathrm{l}$ & $11.62 \pm 0.49$ & $12.25 \pm 0.06$ & $12.01 \pm 0.63$ & $11.82 \pm 0.72$ & $10.85 \pm 0.37$ & $\mathrm{NS}$ \\
\hline Lymphocyte \% & $77.95 \pm 0.05$ & $75.06 \pm 1.21$ & $77.00 \pm 1.68$ & $76.50 \pm 0.64$ & $77.50 \pm 0.28$ & NS \\
\hline Monocyte \% & $4.75 \pm 0.25^{\mathrm{a}}$ & $3.75 \pm 0.25^{\mathrm{b}}$ & $4.25 \pm 0.25^{\mathrm{a}}$ & $4.75 \pm 0.25^{\mathrm{a}}$ & $5.00 \pm 0.13^{\mathrm{a}}$ & $* *$ \\
\hline Heterophil \% & $14.30 \pm 0.23^{\mathrm{b}}$ & $21.00 \pm 1.15^{\mathrm{a}}$ & $16.66 \pm 1.66^{b}$ & $15.75 \pm 0.75^{b}$ & $14.75 \pm 0.47^{b}$ & $* *$ \\
\hline Eosinophil \% & $2.25 \pm 0.25$ & $2.00 \pm 0.01$ & $2.00 \pm 0.05$ & $2.00 \pm 0.04$ & $2.00 \pm 0.03$ & NS \\
\hline Basophil \% & $0.75 \pm 0.25$ & $0.00 \pm 0.00$ & $0.75 \pm 0.25$ & $1.00 \pm 0.11$ & $0.75 \pm 0.21$ & $*$ \\
\hline Hemoglobin g/dl & $8.77 \pm 0.39$ & $8.40 \pm 0.29$ & $8.57 \pm 0.57$ & $8.45 \pm 0.14$ & $9.30 \pm 0.10$ & NS \\
\hline PCV \% & $23.55 \pm 1.02$ & $22.62 \pm 0.42$ & $23.20 \pm 0.88$ & $22.82 \pm 0.42$ & $22.46 \pm 1.18$ & NS \\
\hline $\mathrm{RBC} \times 10^{6} / \mu \mathrm{l}$ & $2.19 \pm 0.09$ & $2.07 \pm 0.03$ & $2.22 \pm 0.08$ & $2.02 \pm 0.02$ & $2.06 \pm 0.13$ & NS \\
\hline $\mathrm{MCH}$ & $39.97 \pm 0.48$ & $42.52 \pm 0.71$ & $43.02 \pm 1.51$ & $42.80 \pm 0.80$ & $43.33 \pm 1.01$ & NS \\
\hline $\mathrm{MCV}$ & $107.25 \pm 0.29$ & $108.75 \pm .71$ & $108.85 \pm 0.24$ & $106.85 \pm 1.12$ & $107.76 \pm 1.38$ & NS \\
\hline $\mathrm{MCHC}$ & $37.27 \pm 0.44^{\mathrm{b}}$ & $39.07 \pm 0.61^{\mathrm{ab}}$ & $39.50 \pm 1.30^{\mathrm{ab}}$ & $40.16 \pm 0.71^{\mathrm{a}}$ & $40.16 \pm 0.43^{a}$ & $*$ \\
\hline
\end{tabular}

$\overline{\mathrm{a}, \mathrm{b},}=$ Mean values within rows with different superscripts letters are significantly different.* $(p<0.05), * *(p<0.01)$ NS non significant. 


\section{Enas K. Aziz and Shimaa R. Masoud}

Table 8: Effect of feeding frequency and amount on biochemical parameters of broiler (Means \pm SE).

\begin{tabular}{|c|c|c|c|c|c|c|}
\hline \multirow[t]{2}{*}{ Items } & \multicolumn{5}{|c|}{ Feeding regimens } & \multirow{2}{*}{$\begin{array}{l}P \\
\text { value }\end{array}$} \\
\hline & $\mathrm{T} 1$ & $\mathrm{~T} 2$ & $\mathrm{~T} 3$ & $\mathrm{~T} 4$ & T5 & \\
\hline Total protein(g\dl) & $3.10 \pm 0.11^{\mathrm{b}}$ & $3.60 \pm 0.05^{\mathrm{a}}$ & $3.34 \pm 0.05^{\mathrm{b}}$ & $3.16 \pm 0.10^{b}$ & $2.82 \pm 0.09^{c}$ & $* *$ \\
\hline Albumin(g\dl) & $1.55 \pm 0.11^{\mathrm{b}}$ & $1.28 \pm 0.05^{\mathrm{c}}$ & $1.85 \pm 0.04^{\mathrm{a}}$ & $1.62 \pm 0.06^{b}$ & $1.63 \pm 0.05^{\mathrm{b}}$ & $* *$ \\
\hline Globulin(g\dl) & $1.54 \pm 0.01^{\mathrm{b}}$ & $2.32 \pm 0.03^{\mathrm{a}}$ & $1.48 \pm 0.02^{\mathrm{b}}$ & $1.53 \pm 0.08^{b}$ & $1.18 \pm 0.01^{\mathrm{c}}$ & $* *$ \\
\hline $\mathrm{A} / \mathrm{G}$ ratio & $1.04 \pm 0.10^{\mathrm{b}}$ & $0.54 \pm 0.02^{\mathrm{c}}$ & $1.23 \pm 0.06^{\mathrm{b}}$ & $1.12 \pm 0.09^{b}$ & $1.57 \pm 0.17^{\mathrm{a}}$ & $* *$ \\
\hline $\begin{array}{l}\text { Gamma } \\
\text { globulin(g\dl) }\end{array}$ & $0.11 \pm 0.01^{b}$ & $0.26 \pm 0.01^{\mathrm{a}}$ & $0.10 \pm 0.04^{b}$ & $0.14 \pm 0.01^{b}$ & $0.09 \pm 0.05^{\mathrm{c}}$ & $* *$ \\
\hline
\end{tabular}

${ }^{a, b, c}=$ Mean values within rows with different superscripts letters are significantly different $* *(\mathrm{p}<0.01)$.

Table 9: Effect of feeding frequency and amount on growth hormone and glucose concentration of broiler (Means \pm SE)

\begin{tabular}{|c|c|c|c|c|c|c|}
\hline \multirow[t]{2}{*}{ Items } & \multicolumn{5}{|c|}{ Feeding regimens } & \multirow{2}{*}{$\begin{array}{l}P \\
\text { value }\end{array}$} \\
\hline & $\mathrm{T} 1$ & $\mathrm{~T} 2$ & T3 & $\mathrm{T} 4$ & $\mathrm{~T} 5$ & \\
\hline Glucose (mg\dl) & $142.75 \pm 6.65^{b}$ & $152.60 \pm 11.72^{\mathrm{a}}$ & $140.20 \pm 7.25^{\mathrm{b}}$ & $131.80 \pm 10.77^{\mathrm{c}}$ & $135.60 \pm 5.72^{\mathrm{bc}}$ & $*$ \\
\hline $\begin{array}{l}\text { Growth hormone } \\
(n g \backslash m \mathrm{l})\end{array}$ & $4.72 \pm 0.27^{\mathrm{c}}$ & $7.40 \pm 0.17^{\mathrm{a}}$ & $4.12 \pm 0.15^{\mathrm{c}}$ & $3.76 \pm 0.32^{\mathrm{c}}$ & $5.72 \pm 0.51^{\mathrm{b}}$ & $* *$ \\
\hline
\end{tabular}

\section{DISCUSSION}

In this study, offering two equal feeding per day was significant improved overall body weight, body weight gain, and average daily gain and feed intake, however the FCR of broiler chicks was not affected. These results might be attributed to offering two equal feeding for chicks per day at 6:30 and 18:30 increased feed intake during cooler temperatures at this time, which allows the chicks to meet their nutrient requirements for growth (Abdul Azis and Afriani, 2017). Besides, feeding frequency could reflect changes in the pattern of circulating hormones such as growth hormone as listed before in result section, that may result from pulsate feed intake (Su et al., 1999). The presented results were in agreement with Chris $\boldsymbol{e t}$ al., (2011) who reported that feeding chicks more than once a day increased growth performance. Farghly and Hassanien, (2012) stated that body weight gain of birds was significantly affected by different feeding frequencies.

Liu et al., (2020) reported geese fed 3 times daily had a lower body weight, average daily gain from 28 to 41 day of age compared with those of the other groups but at 56 to 69 day of age had a higher average daily gain than those of the ad libitum group and those fed 4 times daily. In contrast, Tolkamp et al., (2005) reported that there were no effects of restriction feed either as nutritional quality or quantity or time accessing on body weight and body weight gain. Adikari et al., (2018) concluded that lack of significant effect of feeding frequency to live body weight gain and FCR of broiler chickens. Saber et al., (2011) and Jones and Farrell, (1992) reported that FCR was not affected with changing of feeding frequencies.
These differences in the findings may be due to the difference in the methods of feed restriction or the duration and time of feed frequency. The higher feed intake could be attributed to increased feeding frequency improved the feed utilization through improvements in nutrient digestibility (Fanimo et al., 2003). As well as improving the ability of chicks to compensate for feeding two meal or three meals compared to ad libitum fed chicks over time (Farghly and Hassanien, 2012). The results of present study agreed with the findings of Ozkan et al., (2003) and Bley and Bessei, (2008) who reported that increasing feeding frequency for broiler had a significant effect on feed intake and feed efficiency. Chris et al., (2011) concluded that feeding twice and triple daily feeding increased feed consumption than once or more than triple daily.

Feeding frequency and amount had a significant effect on chick's behavior Increasing the feeding behaviour of chicks under feeding frequency could be attributed to increasing feed intake of chicks that reflect on body weight as presented and discussed previously. Moreover, increasing frequency of preening behavior might indicate increasing the behavioural activities of the bird. Duncan and WoodGush, (1972) thought that preening behaviour is considered essential both in response to stimulation from feather disorder and as displacement activity in mild frustrating or conflict situations. Lefebvre, (1982) reported that birds perform preening activity rhythmicity in an undisturbed environment. 
Chicks of $5^{\text {th }}$ program were exhibited significantly higher foraging behavior than those of $3^{\text {rd }}$ and $4^{\text {th }}$ program. Tahamtani and Riber, (2020) reported the birds likely use foraging and exploration as approaches to control stress caused by food shortage. Chicks of $5^{\text {th }}$ program showed less feeding than other programs so that increase foraging to compensate their nutrient requirement.

The welfare related behavior such as fear, and stress indicated by the duration of tonic immobility test (Campo et al., 2005; Ghareeb et al., 2008). The duration of tonic immobility considered behavioral index of fear (Faure and Mills, 1998). This study reported that feeding frequency decreased the duration of TI test. These results indicated the availability of feed two times or more per day for broiler chicks might decrease stress effect and improve welfare and performance. Jones, (1986); Scott and Moran, (1993) reported that long duration of TI is revealed the bird is more frightened and more fearful, and a short duration is indicative of low levels of fearfulness.

Offering two equal feeding daily (T2) for chicks improved percentage of carcass and spleen. These results supported by improved body weight, weight gain and chick immunity under this feeding program (T2). These results were disagreed with Farghly and Hassanien, (2012) who showed that feeding frequency of broiler chicks had no significant effect on percentage of dressed carcass, heart, gizzard, and intestine. Similar results were observed in geese by Liu et al., (2020) who concluded that carcass traits at 70 day of geese age were not affected by feeding frequency. Farghly and Makled, (2015) and Farghly et al., (2019) indicated that intermittent feeding did not affect the carcass characteristics and organ weights of broilers except for the heart.

However, Aliakbarpour et al. (2013) observed a significant decrease in carcass percentage when fed broiler chickens 5 times daily. Nematallah $\boldsymbol{e t}$ al., (2003) indicated that restricted feeding of Muscovy ducklings did not affect the carcass characteristics and the relative weights of different organs, except the relative weight of liver.

The results of this study revealed that the feeding frequency had no significant effect on hematological parameters as $\mathrm{Hb}, \mathrm{PCV}, \mathrm{RBCs}, \mathrm{MCH}$, and MCV. On the other hand offering two equal feeding per day improved the immunity of chicks through increasing heterophil level, total protein, globulin, and gamma globulin levels, as well as a lower $\mathrm{A} / \mathrm{G}$ ratio than the other programs.

However, feed restriction had only a little impact on blood variables, our data revealed that, feed frequency as one of feed restriction strategy improved birds immunity through increasing growth hormone, which stimulates the immune system. This could be because growth hormone induces a rapid rise in a variety of immune cells, including WBCs, and so improves the immune system's overall efficacy. Additionally, thymus gland and spleen, as well as lymphocyte proliferation and differentiation and bone marrow function, all of this require GH (Wikipedia, 2021).

Results of this study concur with Fayed $\boldsymbol{e t}$ al., (2012) who found that birds subjected to dietary restriction without enzyme supplementation displayed a clear heterophilia in terms of physiological responses. Our findings backed up Kidd, (2004) who reported that diet regimens increase immunity and reduce illness severity in broiler chickens. Additionally feed restriction augmented the immunological response of broilers that had been damaged by heat stress (Khajavi et al., 2003). Our results contradict those of Shabani et al., (2015); Davood-Omam et al., (2019) who found that feed limitation had a very limited effect on broiler chicken immunity. Furthermore, Rahimi et al., (2015) found that a feed restriction had no effect on broiler chicken immunity.

The feed restriction had a very limited effect on blood variables, indicating that the nutritional and metabolic status of broiler chickens remained unchanged even in the treated groups, which is consistent with Junqueira et al., (2003) who indicated that feed restriction has no effect on haematological parameters $(\mathrm{He}, \mathrm{Hb}, \mathrm{Ht}, \mathrm{MCV}, \mathrm{MCH}$, MCHC). Davoodi-Omam et al., (2019) who found that feed limits had no effect on broiler chicken blood variables. When Maxwell et al., (1990) studied the influence of diet restriction on erythrocyte characteristics and reported significant alterations in $\mathrm{PCV}, \mathrm{RBCs}, \mathrm{Hb}, \mathrm{MCV}$, and MCHC that might attributed to using different feeding regime.

Besides, offering feed to chicks twice per day with equal amount improved serum glucose and growth hormone that indicated improving weight and weight gain of chicks as presented previously. This owing to the action of growth hormone, which reduces glucose uptake in the liver and promotes gluconeogenesis and stimulates protein anabolism in many tissues. This effect reflects increased amino acid uptake, protein synthesis and decreased oxidation of proteins (Wikipedia, 2021). Also Increases muscle mass through sarcomere hypertrophy. Our findings are consistent with those of Fayed et al., (2012), who found that broiler chicken subjected to diet restriction without enzyme supplementation had the highest overall mean blood glucose level. 
Our findings contradict those of Dewil $\boldsymbol{e t}$ al., (1999); Kubíková et al., (2001) and Rajman et al., (2006) who found no effect of quantitative feed limitation on blood glucose levels on broiler chicken.

\section{CONCLUSION}

It can be concluded that, the feeding frequency with different amount especially two equal feeding per day could be a desirable feeding approach that might produce good healthy birds with maximum growth performance and improved welfare.

\section{ACKNOWLEDGMENTS}

We are grateful to all staff members of Department of Husbandry and Animal Wealth Development and Physiology Department, Faculty of Veterinary Medicine, University of Sadat City for providing help in the study.

\section{Statement of conflict of interests} interest.

All authors declare there is no conflict of

\section{REFERENCES}

ABDELBASIT, B.H., ABDELRAHMIN, A.M., AHMED, M.E., EGBAL,S.A. AND ABUBAKER, A.A., 2016. Effect of feed supplemented with xylam enzyme on performance, carcass characteristics and meat quality of broiler chicks. J. App. Vet. Sci, 1(1):1520.https://dx.doi.org/10.21608/javs.2016.61822

ABDUL AZIS. AND AFRIANI, 2017. Effect of feeding time restriction during the growing period on growth performance of broiler chickens. Asian J. Poult. Sci., 11: 70-74.

ADEREMI, F.A., 2004. Effects of replacement of wheat bran with cassava root sieviate supplemewnted or unsupplemented with enzyme on the haematology and serum biochemistry of pullet chicks. Trop J Anim Sci.;7:147-153.

ADIKARI, A., NANDASENA, W., NAYANANJALIE, W. AND JAYATHILAKA, B., 2018. Effects of feeding frequency on fat deposition and growth Performance in broiler chickens. International Journal of Liv. Res, 8 (9), 62-72. Doi 10.5455 /ijlr. 0324050424.

ALIAKBARPOUR, H. R., CHAMANI, M., RAHIMI, G., SADEGHI, A. A. AND QUJEQ, D., 2013. Intermittent feeding programme and addition of Bacillus subtilis based probiotics to the diet of growing broiler chickens: influence on growth, hepatic enzymes and serum lipid metabolites profile. Arch. Anim. Breed. 56:410-422.

AMAKIRI, A.O., OWEN, O.J. AND JACK, D.O., 2009. Effects of refined petroleum product (kerosene) flame and fumes on haematological characteristics of broiler chickens. Proc of the 34th Annual Conf of the Nig Soc for Anim Prod.;41-44.

BARACHO, M., CAMARgo, G., LIMA, A., MENTEM, J., MOURA, D. AND MOREIRA, J., NÄÄS, I., 2006. Variables impacting poultry meat quality from production to pre-Slaughter: A Review. Revis Bras de Ciên Avíc., 8, 201-212.

BLEY, T.A. AND BESSEI, W., 2008. Recording of individual feed intake and feeding behavior of pekin ducks kept in groups. Poult. Sci, 87, 215-221.

BUTZEN, F. M., RIBEIRO, A. M. L., VIEIRA, M. M., KESSLER, A. M., DADALT, J. C. AND DELLA, M. P., 2013. Early feed restriction in broilers. performance, body fraction weights, and meat quality. J. Appl. Poult. Res. 22:251-259.

CAMPO, J.L., GIL, M.G., DAVILA, S.G. AND MUNOZ, I., 2005. Influence of perches and footpad dermatitis on tonic immobility and heterophil to lymphocyte ratio of chickens. Poult Sci 84:1004-1009.

CHRIS, B., NDOME, A.O., EKWU. AND ALFRED, A., 2011. Effect of feeding frequency on feed consumption, growth and feed conversion of Clarias gariepinus \% X Heterobranchus longifilis \& Hybrids. Amer-Eura. J. of Sci. Reser 6: 06-12.

DAVOOD I-OMAM, M., DADAS HBEIKI, M., CORAZZIN, M. AND SEIDA VI, A., 2019. Effect of feed restrictions on performance, blood variables and immunity of broiler chickens. Vet. arhiv 89, 71-86.

DE JONG, I. C., VAN VOORST, A. S. AND BLKHUIS, H. J., 2003. Parameters for quantification of hunger in broiler breeders. Physiol. Behav. 78(4-5):773-783.

DEWIL, E., DARRAS, V.M., SPENCER, G.S.G., LAUTERIO, T.J. AND DECUYPERE, E., 1999. The regulation of $\mathrm{GH}$ dependent hormones and enzymes after feed restriction in dwarf and control chickens. Life Sci., 1999; 64: 1359-1371.

DUNKAN, I.J.H. AND WOOD-GUSH, D.G.M., 1972: An analysis of displacement preening in the domestic fowl. Anim. Behav. 20, 68-71.

FANIMO, A.O., ODUGUWA, O.O., ADESEHINWA, A.O.K., OWOEYE, E.Y. AND BABATUNDE, O.S., 2003. Response of weaner pigs to feed rationing and frequency of feeding. Live. Res. for Rural Develop, 15.

FRASER, A.F. AND BROOM, D.M., 1990. Farm animal behavior and welfare. 3rd Ed ,.Bailliere Tindall.

FARGHLY, M. AND HASSANIEN, H.M., 2012. Effect of feed frequencies and durations on performance of broiler chicks. Egypt. Poult. Sci. Vol (32) (II): (273288).

FARGHLY, M. F. A. AND MAKLED, M. N., 2015. Application of intermittent feeding and flash lighting regimens in broiler chickens management. Egypt. J. Nutr. Feeds 18:261-276.

FARGHLY, M. F., MAHROSE, K. M., AHMAD, E. A. M. AND REHMAN, Z. U., YU S., 2019. Implementation of different feeding regimes and flashing light in broiler chicks. Poult. Sci. 98:20342042.

FAURE, J.M. AND MILLS. A., 1998. Improving the adaptability of animals by selection. In: T. Grandin (Ed.), Genetics and the Behavior, Academic Press, San Diego, pp: 235-264. 
FAYED, R. H., ABEER, H. A. R. AND BASSMA, M. B., 2012. Welfare assessment of broiler chickens subjected to feed restriction and fed enzyme supplemented diet. J. Ameri Sci; 8 (12).

GHAREEB, K., NIEBUHR, K., AWAD, W.A., WAIBLINGER, S. AND TROXLER, J., 2008. Stability of fear and sociality in two strains of laying hens. Br Poult Sci 49: 502-508.

JONES, R.B., 1986. The tonic immobility reaction of the domestic fowl: a review. World's Poult Sci. Journal, 42: 82-96.

JONES, G.P. AND FARRELL, D.J., 1992. Early-life food restriction of broiler chickens. i. methods of application, amino acid supplementation and the age at which restrictions should commence. Brit. Poult. Sci, 33, 579-587.

JUNQUEIRA, O.M., FONSECA, L.E.C., ARAÚJO, L.F., DUARTE, K.F., ARAÚJO, C.S. DA S. AND RODRIGUES, E.A.P., 2003. Feed restriction on performance and blood parameters of broilers fed diets with different sodium levels. Rev. Bras. Cienc. Avic. 5: 2.

KHAJAVI, M., RAHIMI, S., HASSAN, Z. M., KAMALI, M. A. AND MOUSAVI, T., 2003. Effect of feed restriction early in life on humoral and cellular immunity of two commercial broiler strains under heat stress conditions. Brit. Poult. Sci, 44(3): 490-497.

KHURSHID, A., KHAN, A. A., BANDAY, M. T., GANAI, A. M., KHAN, H. M., CHOUDHARY, A. R., ADIL, S., MANZOOR, A., AFZAL I. AND UNTOO, M., 2019. Effect of feed restriction on performance of broiler chicken. J. Entomol. Zool. Stud. 7:1054-1056.

KIDD, M. T. 2004. Nutritional modulation of immune function in broilers poultry science 83:650-657.

KUBÍKOVÁ, L., VÝBOH, P. AND KOŠTÁL, L., 2001. Behavioural endocrine and metabolic effects of food restriction in broiler breeder hens, Acta Vet. Brno ; 70: 247-257.

LEFEBVRE, L., 1982. The organization of grooming in budgerigars. Behav. Proc. 7: 93-106.

LIU, J. B., CAO, S.C., YANG, Y. AND ZHANG, H. F., 2019. Effects of meal frequency on growth performance and muscle protein synthesis in suckling piglets. Chin. J. Anim. Nutr. 31:1-9.

LIU, Z. L., XUE, J. J., HUANG, X. F., LUO, Y., LIANG, M. R., LI, C. J., WANG, Q. G. AND WANG, C., 2020. Effect of feeding frequency on the growth performance, carcass traits, and apparent nutrient digestibility in geese. Poult. Sci. 99:4818.

MAXWELL, M.H., ROBERTSON, G.W., SPENCE, S. AND MCCORQUODALE, C.C., 1990. Comparison of hematological values in restricted and ad libitum fed domestic fowls: red blood cell characteristics. Brit. Poult. Sci. 31: 407-413.

MELUZZI, A., PRIMICERI, G., GIORDANI, R. A. AND FABRIS, G., 1992. Determination of blood constituents reference values in broilers, Poult. Sci. 71, 337-345.

MOHAMMADALIPOUR, R., RAHMANI, H. R., JAHANIAN, R., RIASI, A., MOHAMMAD ALIPOUR, M. AND NILI, N., 2017. Effect of early feed restriction on physiological responses, performance and ascites incidence in broiler chickens raised in normal or cold environment. Animal 11:219-226.

NEMATALLAH, G.M.A., FATEN, A.A.I. AND ELKAITY, A.M., 2003. The influence of early food restriction on performance, immunoresponsiveness and some physiological variables in Muscovy ducklings. Egypt. Poult. Sci., 23: 355-373.

NEVES, D., BANHAZI, T. AND NÄÄS, I., 2014. Feeding behaviour of broiler chickens: a review on the biomechanical characteristics. Revista Brasileira de Ciência Avícola, 16, 01-16.

OLUKOMAIYA, O.O., ADEYEMI, O.A., SOGUNLE, O.M., ABIOJA, M.O., IWUCHUKWU, P.O. AND EMUVEYAN, U., 2014 Effects of feed restriction and ascorbic acid supplementation on haematological parameters of Marshall broiler chickens., Indian Journal of Innovations and Developments Vol 3.P.Emuveyan: (2), ISSN: 2277-5390. OZKAN, S., AKBAS, Y., ALTAN, O., ALTAN, A., AYHAN, V. AND OZKAN, K., 2003. The effect of short-term fasting on performance traits and rectal temperature of broilers during the summer season. Brit. Poult. Sci, 44, 88-95.

PETER, R. F. AND GERNAT, A. G., 2006. Factors that affect feed intake of meat birds: A Review. Inter. J. of Poult. Sci., 5: 905-911.

RAHIMI, S., SEIDAVI, A., SAHRAEI, M., BLANCO, F. P., SCHIAVONE, A. AND MARTINEZ MARIN, A. L., 2015. Effect of feed restriction and diet nutrient density during re-alimenatation on growth performance, carcass traits, organ weight, blood parameters and the immune response of broilers. Ital. J. Anim. Sci. 14, 4037.

RAJMAN, M., JURÁNI M., LAMOSOVÁ, D., MÁCAJOVÁ, M., SEDLACKOVÁ, M., KOST'ÁL, L., JEZOVÁ, D. AND VÝBOH, P., 2006. The effects of feed restriction on plasma biochemistry in growing meat type chickens (Gallus gallus). Comp Biochem Physiol A Mol Integr Physiol. 2006;145(3): 363-71.

SABER, S.N., MAHERI-SIS, N., TELLI, A.S., HATEFINEZHAD, K., GORBANI, A. AND YOUSEFI, J., 2011. Effect of feed restriction on growth performance of broiler chickens. Anna. of Bio. Res, 2, 247-252.

SAIED, J.M., AL-JABARY, Q.H. AND THALIJ, K.M., 2011. Effect of dietary supplement yeast culture on production performance and hematological parameters in broiler chicks. Interna. Journal of Poult. Sci. 10, pp.376- 380 .

SCHALM, O.W., 1986. Veterinary hematology. The Pig: Normal hematology with comments on response to disease. 4th edition. Lea and Febiger, Philadelphia, pp.523.

SCOTT, G.B. AND MORAN, P., 1993. Fear levels in laying hens carried by hand and by mechanical conveyors. Appl. Anim. Behav. Sci., 36: 337- 346.

SHABANI, S., SEIDAVI, A., ASADPOUR, L. AND CORAZZIN, M., 2015. Effects of physical form of diet and intensity and duration of feed restriction on the growth performance, blood variables, microbial flora, immunity, and carcass and organ characteristics of broiler chickens. Livest. Sci. 180, 150-157. 
SU, G., SORENSEN, P. AND KESTIN, S. C., 1999. Meal feeding is more effective than early feed restriction. at reducing the prevalence of leg weakness in broiler chickens. Poult. Sci., 78:949-955.

TAHAMTANIA, F. M. AND RIBER, A. B., 2020. The effect of qualitative feed restriction in broiler breeder pullets on fear and motivation to explore. Appl. Anim. Behav. Sci. 228, 105009.

TOLKAMP, B., SANDILANDS, J. V., KYRIAZAKIS, 2005. Effects of qualitative feed restriction during rearing on the performance of broiler breeders during rearing and lay. Poult. Sci., 84:1286-1293.

WIKIPEDIA, 2021. Growth hormone. https://en.wikipedia.org/wiki/growth hormone.
How to cite this article:

Enas K. Aziz and Shimaa R. Masoud, 2021.The Effect of Feeding Frequency and Amount on Performance, Behavior and Physiological Responses of Broilers. Journal of Applied Veterinary Sciences, 6 (3): $76-85$. DOI:https://dx.doi.org/10.21608/javs.2021.80537.1086 